\title{
La organización de la respuesta: innovación y recuperación en las salas de operaciones financieras del Bajo Manhattan
}

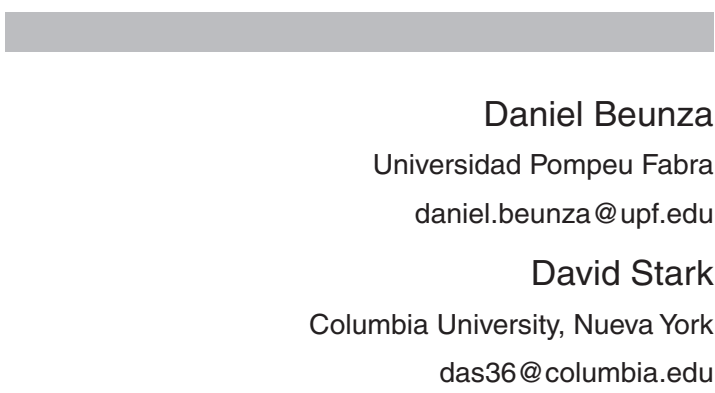

RESUMEN

¿Cuál es la base organizativa de la capacidad de recuperación de una crisis? En este ensayo examinamos una sala de operaciones financieras dañada en el ataque al World Trade Center el 11 de septiembre de 2001. ¿Qué revela la crisis sobre las prácticas sociales y los instrumentos tecnológicos de compra-venta? A partir de nuestra investigación de campo anterior al once de septiembre, mostramos cómo la organización heterárquica (opuesta a la jerárquica) de la sala de operaciones promovió la innovación espontáneamente, a medida que los acontecimientos se desarrollaban. A través de nuestras observaciones posteriores al ataque, así como unos grupos de enfoque con ejecutivos de varias empresas financieras del World Trade Center, mostramos que las características heterárquicas contribuyeron a la innovación en la respuesta a la crisis. En condiciones de incertidumbre radical no pueden conocerse de antemano los recursos que serán necesarios, ni siquiera saber por adelantado qué podría ser un recurso. La inteligencia distribuida lateralmente y la tolerancia de múltiples registros de evaluación e interacción proporcionan estructuras generativas en las que la redundancia replicativa de la planificación de emergencias se enfrenta a sus límites. Finalizamos con una breve discusión de las implicaciones que nuestros hallazgos sobre innovación, ubicación y capacidad de respuesta tienen para los actuales cambios en la geografía urbana de las finanzas en el Bajo Manhattan.

Palabras clave: Teoría de Organizaciones, Heterarquía, Planificación de Emergencias, Redes Sociotécnicas, Geografía Económica, Once de Septiembre [de 2001]. 


\section{INTRODUCCIÓN}

En este ensayo examinamos una sala de operaciones financieras que fue destruida en el ataque del once de septiembre al World Trade Center (WTC). La sala de operaciones, parte de un importante banco de inversiones internacional, estaba situada en el World Financial Center, adyacente al WTC. Tras el ataque del once de septiembre, el equipo directivo de la sala de operaciones se reagrupó en un centro de emergencia en Nueva Jersey y estimó que se necesitarían de tres semanas a tres meses para reanudar las operaciones. Sin embargo, tan sólo seis días después, cuando los mercados financieros volvieron a abrir (17 de septiembre de 2001), estos traders estaban operando de nuevo ${ }^{1}$. ¿Qué clase de organización hizo posible tal recuperación? ¿Qué revela la crisis sobre prácticas sociales y tecnología financiera? ¿Y qué implica esta organización de la innovación para la recuperación del Bajo Manhattan en su conjunto?

Somos afortunados de poder abordar estas cuestiones sobre la base de una íntima familiaridad con las prácticas de una sala de operaciones en Wall Street. Dos años antes del 11 de septiembre de 2001, comenzamos una investigación etnográfica en una sala de operaciones. El proyecto era parte de un proyecto de estudio de cómo estaba cambiando la organización de Wall Street en respuesta a las nuevas tecnologías de la información. ¿Cuál es, nos preguntábamos, el papel de la ubicación en condiciones de conectividad global? Nuestros descubrimientos, sintetizados en la primera sección del artículo, son paradójicos: cuanto más se dispone de información en tiempo real y de forma simultánea para todos los actores del mercado, más se desplaza la ventaja de economías de la información hacia procesos de interpretación. La sala de operaciones, tan abundante en información, es un lugar de interpretación. La innovación es el producto de la interacción a través de principios heterárquicos de evaluación, y ello se produce dentro de la proximidad física que ofrece la sala de operaciones.

A partir del once de septiembre, continuamos nuestra investigación etnográfica siguiendo a los traders en su reubicación en Nueva Jersey. Los descubrimientos que realizamos allí, de los que se da cuenta en la segunda parte del artículo, confirmaron nuestras primeras impresiones de que los traders atribuyen una elevada importancia a la proximidad física. Ésta facilita los tipos de asociaciones financieras que tan importantes resultan para su trabajo. También revelan que el negocio descansa no sólo en la organización social, sino también

\footnotetext{
1 Nota del traductor: No es fácil traducir traders al castellano. A diferencia de otros intermediarios financieros como los brokers o corredores de bolsa, o los salesmen o agentes de ventas, los traders actúan por cuenta propia: además de tomar posiciones para su cliente, también lo hacen para el banco en el que trabajan. Dada la importancia de las diferencias en la tecnología usada por unos y otros para los sucesos descritos en el artículo, se ha mantenido la expresión traders en inglés a lo largo del texto.
} 
en un conjunto complejo de conexiones técnicas. El ataque del once de septiembre dañó la sala de operaciones y trastornó por completo tecnologías fundamentales para el negocio financiero moderno. Ello dejó al descubierto el carácter sociotécnico de estas relaciones: el colapso de la tecnología es la sociedad hecha visible.

Además de nuestras observaciones directas en la reubicada sala de operaciones, en la tercera parte del artículo damos cuenta de nuestras discusiones con altos directivos de otras empresas financieras del complejo del WTC. ¿Cómo pudieron estas empresas responder con tanta rapidez y eficacia, cuando su funcionamiento se había trastornado de forma tan desoladora? En los fragmentos de estas conversaciones reproducidos más adelante, nuestros interlocutores describen el ambiente de miedo y defunción en el que trabajaron en aquellos días terribles, y cuentan cómo los fuertes lazos personales que les unían fueron la clave de la recuperación. Como veremos, la respuesta organizativa se apoyó más en estructuras de autoorganización y coordinación lateral que en planes de emergencia y jerarquías de mando. En resumen, la inteligencia distribuida, tan importante en el funcionamiento cotidiano de las salas de operaciones, formó la base de la respuesta organizativa a la crisis. Mientras que la innovación puede verse como respuesta a la incertidumbre, la respuesta a la crisis puede verse como un ejemplo particular de innovación. Exploramos brevemente la relevancia de este concepto para la época posterior al once de septiembre, cuando la métrica del estado de preparación adquiere un mayor relieve en las valoraciones de las empresas.

Este artículo finaliza con una breve discusión de las consecuencias de nuestros descubrimientos sobre la innovación, localización y respuesta para la reurbanización del Bajo Manhattan. ¿Se extienden las dinámicas espaciales de la sala de operaciones a Wall Street? ¿Sobrevivirá la estructura concéntrica del distrito financiero, centrado en la Bolsa de Nueva York (NYSE, New York Stock Exchange)? Sostenemos que el futuro de Wall Street reside más en redes multicéntricas que en la proximidad al NYSE.

Sin embargo, las decisiones de localización no se toman solamente por factores de transferencia de conocimiento entre comunidades de práctica, sino también por los precios de los alquileres, las características de la infraestructura o del tiempo invertido en los desplazamientos diarios al trabajo. La lógica de la preparación dicta que tras el once de septiembre las empresas deben dispersar sus operaciones, no sólo a través de diferentes edificios, sino también por múltiples redes eléctricas, prefijos telefónicos y los centros de transporte. ¿Cómo están estas nuevas inquietudes sobre seguridad remodelando la geografía urbana de las finanzas? Observamos que mientras que la innovación tiende a acercar a las empresas entre sí, la preocupación por la seguridad tiende a alejarlas. Estas fuerzas opuestas plantean nuevos dilemas a las empresas del sector financiero de Nueva York. 


\section{LOCALIZACIÓN: COMUNIDADES DE PRÁCTICA EN UNA SALA DE OPERACIONES}

La sala de operaciones en renta variable de International Securities (nombre seudónimo), al igual que sus homólogos en el Trade Center, ofrece un claro contraste con la Norteamérica corporativa. Entre usted en el complejo de oficinas del World Financial Center. Tome el ascensor y suba a una de sus torres. A la salida del ascensor del piso veinte se abre un mar de mesas con pantallas Bloomberg de múltiples colores. Las mesas están ocupadas por traders vestidos de manera informal. A diferencia de una oficina estándar con cubículos y diseño orientado a subrayar las diferencias de jerarquía, las salas de operaciones son superficies de planta abierta en las que la información fluye libremente ${ }^{2}$. En lugar de alojar al personal administrativo en el centro de la planta, como hacen las corporaciones clásicas, International Securities sitúa a su gerente en el centro, al alcance de todos. Y en lugar de tener a sus altos directivos dispersos por oficinas con ventanal y vistas al exterior (donde la probabilidad de topar con ellos se reduce al mínimo), el banco los pone en la misma mesa que los miembros del equipo que trabaja con ellos, permitiendo a estos últimos acceder a ellos con tan sólo un movimiento de la cabeza o la mano. Recalcando la importancia de la confianza y de la sociabilidad, el banco ha limitado el número de personas en la sala a 150 empleados y tiene una política de «pantallas bajas», para que los miembros de la organización puedan verse unos a otros.

¿Qué hay de los traders, esos privilegiados habitantes de la sala de operaciones? Sus perspectivas y personalidad se han visto radicalmente alteradas por una silenciosa revolución tecnológica que ha barrido Wall Street durante las dos últimas décadas. Esta revolución -la revolución de las finanzas cuantitativas - se inició con el auge de los derivados como los futuros y las opciones, las fórmulas matemáticas como Black-Scholes, la conectividad en red a mercados electrónicos como el NASDAQ y la aparición de ordenadores de alta potencia. En consecuencia, las finanzas se han vuelto matemáticas, interconectadas, informatizadas e intensivas en conocimiento. La simple renta variable (acciones) ya no es de interés para los traders profesionales: en enero de 2000, por ejemplo, la cantidad total de contratos de derivados por pagar en todo el mundo fue de 108 billones de dólares, el equivalente a casi 18.000 dólares por cada ser humano de la tierra (MacKenzie y Millo, 2003). En este contexto, los traders han evolucionado con el sector. Mientras que los traders de la década de los ochenta, descritos por Tom Wolfe (1987) como «Másters del Universo», se caracterizaban por su riqueza, bravuconería y escasa consideración por el pequeño inversor, los actuales traders cuantitativos tienen MBAs en finanzas y doctorados en física y estadística, y hay que entenderlos más bien como ingenieros. Ninguno de ellos usa tirantes.

2 Gladwell (2000) discute varias instancias en que arquitectura y forma organizativa se encuentran estrechamente entrelazadas. Por ejemplo, describe una empresa publicitaria en California que ha recreado por completo la geografía de un pueblo en su oficina central, con calles, plazas y alrededores. 
La estrategia primordial usada por los traders cuantitativos es el arbitraje en sus diferentes combinaciones y estilos (para un tratamiento detallado de valoración y arbitraje, véase Beunza y Stark, 2002) ${ }^{3}$. El arbitraje reside en la posibilidad de interpretar el valor de diferentes maneras, y genera beneficios asociando mercados previamente dispares. Por ejemplo, los arbitradores asocian los mercados de acciones de dos empresas diferentes en proceso de fusionarse, ya que la fusión hace que su valor sea momentáneamente comparable. O asocian las acciones de dos empresas que están en el mismo índice, y por tanto evolucionan de modo semejante; o una acción y una obligación de la misma empresa cuyos valores están relacionados por una cláusula legal que hace que la obligación sea convertible en acción. La esencia del arbitraje está en evitar la vía convencional de valorar una empresa por su valor intrínseco o por el grado de especulación existente en el mercado, y elegir en su lugar una empresa que genere una oportunidad, una valoración nueva y original que se diferencie del valor que el mercado asigna. De este modo, como una metáfora literaria, una operación de arbitraje atrapa y asocia el valor de una acción a alguna otra diferente. Las dos acciones utilizadas para el arbitraje tienen que ser lo bastante semejantes para cubrir el riesgo, pero lo bastante diferentes para que otros traders no hayan visto la semejanza y hayan aprovechado la oportunidad con antelación. Por ello, cada operación es única y diferente de la anterior. Mientras que estrategias financieras convencionales como la búsqueda del valor intrínseco o de la tendencia dependen de un rápido acceso a la información, el arbitraje realiza una interpretación novedosa del valor de una acción. Y mientras que la búsqueda del valor intrínseco es esencialista y la de la tendencia es extrínseca, el arbitraje es asociativo (para una discusión más amplia de esta distinción, véase Beunza y Stark, 2002).

El desafío cognitivo al que se enfrentan nuestros traders es el problema del reconocimiento. Por una parte, deben, por supuesto, ser hábiles para reconocer patrones (por ejemplo, qué datos encajan en los modelos, etc.). Pero si únicamente hubieran de identificar patrones familiares dentro de categorías mentales existentes, no serían innovadores (Brown, 1997; Brown y Duguid, 1998; Clippinger, 1999). La innovación requiere otro proceso cognitivo, que podemos concebir como de re-conocimiento: efectuar asociaciones imprevistas, reinterpretar la situación y evitar quedar encerrado dentro de ella. Ello implica una búsqueda diferente, en la que no se sabe lo que se está buscando pero se reconocerá cuando se encuentre. Una búsqueda distinta de aquella que se da cuando se conocen las coordena-

\footnotetext{
3 El campo emergente de "estudios sociales de las finanzas» reúne a los científicos sociales con un interés en los mercados de capital (los estudios clásicos incluyen a Smith, 1990; Abolafia, 1996) junto con los sociólogos que están interesados en la producción de conocimiento (del campo de los estudios sobre ciencia y tecnología, véanse especialmente MacKenzie y Millo, 2003; Knorr Cetina y Bruegger, 2002; D. MacKenzie, 2004). Otras contribuciones recientes incluyen a Uzzi (1999), Zuckerman (1999), Lepinay y Rousseau (2000), Lepinay (2002), Muniesa (2002), Preda (2002), Riles (2002), Scout y Barrett (2002) y Zaloom (2002).
} 
das de un objetivo conocido, y distinta de buscar un número de teléfono, código de producto o localizador de documento para una entidad o categoría previamente identificada.

La sala de operaciones está equipada para enfrentarse al doble desafío de explotar conocimientos (reconocimiento del modelo) y explorar nuevos conocimientos simultáneamente (prácticas de re-conocimiento) ${ }^{4}$. Cada mesa (por ejemplo, la de arbitraje de fusiones, arbitraje de índices, etc.) se organiza en torno a un principio valorativo y sus correspondientes marcos, métrica e instrumentos ópticos para el reconocimiento de patrones. Es decir, la sala de operaciones es lugar de encuentro de principios de valoración diversos y rivales. Y es la interacción a través de esta heterogeneidad lo que genera la innovación. En lugar de ser burocráticamente jerárquica, la sala de operaciones es heterárquica (para una discusión más amplia sobre este término, ver Stark, 1999; Girard y Stark, 2002). En lugar de relaciones verticales, encontramos relaciones horizontales de pensamiento lateral distribuido; en lugar de una única métrica de valoración, hallamos múltiples métricas de valor; y en lugar de investigación y desarrollo diseñados y dirigidos, encontramos innovación como combinatoria (Kogut y Zander, 1992), es decir, surgida de la interacción entre estos principios e instrumentos. La sala de operaciones distribuye la inteligencia y organiza la diversidad.

Así, en International Securities conviven una variedad de estilos de arbitraje. Cada escritorio de la sala pertenece a una comunidad de práctica distinta (Wenger, 1998; Brown y Duguid, 2000; Orlikowski, 2002), correspondiente a una estrategia diferente, como son el arbitraje de fusiones, el arbitraje de índices o el arbitraje con las órdenes de los clientes. Pero las diferencias entre las mesas no son meramente operativas: diferentes mesas tienen diferentes principios de valoración y una instrumentación diferente (para detalles, véase Beunza y Stark, 2003). Los traders de la mesa de arbitraje de fusiones, por ejemplo, valoran las empresas que son blanco de una OPA («oferta pública de acciones») en términos de la cotización de la empresa que está iniciando dicha OPA. Estos traders se especializan en preguntarse a sí mismos: «¿Cuál es la probabilidad de que las empresas $\mathrm{X}$ e $\mathrm{Y}$ vayan a fusionarse?». Desde el punto de vista analítico, para ellos las empresas son poco más que potenciales autores y blancos de adquisiciones. En cambio, los traders de la mesa de arbitraje de bonos convertibles explotan el valor de las cláusulas de convertibilidad inscritas en algunos bonos, que dan a sus tenedores la opción de convertirlos en acciones. Los traders valoran las acciones como si fueran bonos, y se centran en información que normalmente interesaría sólo a tenedores de bonos. Los traders de la mesa de arbitraje con órdenes de clientes, por ejemplo, ejecutan las órdenes de compra-venta que les dictan sus clientes y,

4 Reinterpretamos el problema de explotación/exploración del aprendizaje organizativo, de March (1991), a través de la óptica del problema de reconocimiento. Sobre un desafío diferente pero relacionado en la industria de los medios, véase Girard y Stark (2002). 
en ocasiones, también idean y sugieren estrategias para estos clientes. Son sociables y cordiales, y comparten salazón de carne (beef jerky), que mascan mientras compran y venden y hablan por teléfono. El sonido de sus voces al teléfono da al resto de la sala acceso al nivel de ansiedad de sus clientes y, de esa forma, a la opinión del mercado en general.

Las asociaciones financieras establecidas por el arbitraje vienen condicionadas por las relaciones sociales en la sala. Cada estrategia de arbitraje asocia acciones de empresas que comparten una propiedad que las hace comparables, tales como la convertibilidad, la volatilidad, la participación en una fusión, la liquidez o la «opcionabilidad». Dado que, como se observó antes, cada mesa de la sala se corresponde a una estrategia diferente, la interacción entre mesas ayuda a los traders a desagregar el valor de una acción o propiedad en sus aspectos o propiedades constituyentes. Una operación de arbitraje de fusiones, por ejemplo, asocia dos acciones que comparten una propiedad — una elevada probabilidad de fusión- pero que puede verse afectada por otra propiedad diferente, como una excesiva volatilidad. La proximidad física a otras mesas ayuda a los arbitradores de fusiones a aislar la propiedad de interés de las no deseadas. Así, por ejemplo, a los traders en arbitraje de fusiones, oír por casualidad a los traders de arbitraje en bonos convertibles puede hacerles conscientes de problemas de convertibilidad importantes para su trabajo. A su vez, los traders de la mesa de arbitraje de bonos convertibles pueden beneficiarse de oír por casualidad los detalles de la volatilidad de una acción que comentan los traders de la mesa de arbitraje de opciones.

Compartir un espacio común también permite a los traders combinar las estrategias desarrolladas por las diferentes mesas en operaciones originales e innovadoras ${ }^{5}$, dando lugar a estrategias sintéticas. En International Securities, por ejemplo, hay una mesa llamada «de situaciones especiales». Sus traders diseñaron recientemente una innovadora "operación de elección» imaginándose a sí mismos como traders en arbitraje de fusiones en un caso que implicaba un intercambio (o swap) de acciones. Considerar un swap como si fuera una fusión les dio una perspectiva diferente sobre el mismo, factor clave en un sector caracterizado por mercados electrónicos y difusión instantánea de la información. La clave, en este caso, fue su proximidad a la mesa de arbitraje en fusiones. De esta forma, un trader no es

5 El concepto de «lugar de trabajo radicalmente co-localizado», de Olson et al. (2002), se asemeja al nuestro, con una diferencia importante: que las interacciones a través de las mesas de la sala de operaciones no tienen lugar dentro de un proyecto o equipo. Es especialmente relevante la discusión de Galison (1997) sobre la arquitectura de la ciencia (véase también Galison y Thompson, 1999), en que muestra que las innovaciones clave en el Proyecto Manhattan vinieron cuando comunidades separadas de físicos teóricos e ingenieros experimentales (cada una de ellas con epistemologías e instrumentos característicos) fueron reunidas en el mismo edificio en el Rad Lab del Massachusetts Institute of Technology. Esta literatura está, además, apoyada por el análisis cuantitativo de Kraut et al. (2002), que descubrieron que tener los despachos en el mismo pasillo era un indicador significativo de colaboración entre científicos, incluso cuando no pertenecían al mismo campo. 
un pensador aislado y contemplativo, sino que interpreta conocimiento distribuido socialmente a través de diferentes mesas en la sala ${ }^{6}$.

Así pues, al intentar entender el modus operandi de la sala de operaciones vimos que su locus operandi tenía una importancia decisiva. Cuanto más observábamos, menos podíamos suscribir la afirmación de que el comercio electrónico eliminaría la importancia de la localización física. Encontramos que cuanto más virtual era la compra-venta de acciones, más aumentaba la relevancia de la proximidad física, por lo menos al nivel de la élite. Es decir, cuanta más información está simultáneamente disponible para cada actor del mercado, más se desplaza la fuente de ventaja estratégica de esta información hacia procesos sociocognitivos de interpretación (Thrift, 1994, 2000; Brown y Duguid, 2000, 2001; Grabher, 2002a, b). Esta particular sala de operaciones obtiene beneficios - considerablemente mayores que los beneficios medios del sector- no mediante un acceso a mejor o más rápidas fuentes de información, sino cultivando comunidades de interpretación.

Además de procesos cognitivos distribuidos por medio de la ubicación en espacios comunes, la tecnología es otra fuente clave de ventaja competitiva en esta sala de operaciones. International Securities invierte masivamente en terminales Bloomberg. Éstas permiten a los traders representar el valor financiero de múltiples formas diferentes, tales como spread plots, modelos de valoración de bonos, u hojas de cálculo con vínculos activos con el mercado. Las conexiones de banda ancha dan a los traders acceso al mercado con una ventaja temporal decisiva sobre los pequeños inversores, proporcionándoles datos sobre precios casi en tiempo real. Una plataforma informática llamada motor financiero automatiza todas las operaciones administrativas relativas a la compra-venta, tales como registro de las transacciones o la fragmentación de las mismas para evitar que sean descubiertas por los rivales. Y numerosos traders utilizan un sistema informático llamado robot financiero. Estos robots son sistemas constituidos por conexiones, algoritmos y hardware informático que reciben datos del mercado y, a su vez, envían órdenes de compra-venta según algún principio teórico financiero como, por ejemplo, la reversión a la media.

Sin embargo, la ingeniería no da por sí sola ventaja a International Securities sobre sus rivales. La clave reside en la interacción entre la tecnología, los humanos y las ideas, una red sociotécnica constituida por estos tres elementos (Latour, 1991; Callon, 1998; véase

\footnotetext{
6 La noción de pensamiento distribuido fue desarrollada en la obra de Suchman (1987) y Hutchins (1995). Hutchins (1995) demostró que el proceso cognitivo de navegación de un buque de guerra norteamericano se distribuye a través del equipo, sus artefactos y representaciones internas y externas. De modo semejante, Suchman (1987) observó que los sistemas expertos de las fotocopiadoras frecuentemente no logran ayudar a sus usuarios humanos a superar una dificultad, pese a que otro humano presente en el mismo espacio no habría tenido ningún problema en proporcionar el consejo correcto. La razón, según Suchman, es que los sistemas expertos fallan críticamente en constituir inteligibilidad de forma conjunta con el usuario, al no estar preprogramados para la situación que se presenta.
} 
también Lane y Maxfield, 1996, sobre el espacio agente-artefacto). Los robots financieros son un buen ejemplo de esa interacción. Pero entre aquellos cables, chips y líneas de código hay un abundante elemento social. Por ejemplo, el algoritmo de compraventa que utiliza el robot para operar se desarrolla en la llamada pizarra blanca, una sala diseñada para la interacción informal entre programadores y traders. El robot es controlado por un trader humano, llamado trader de arbitraje estadístico, cuyo trabajo consiste en detener el algoritmo siempre que la situación del mercado deje de ser coherente con la teoría que inspira al código. Por ejemplo, cuando dos empresas se fusionan, el principio de reversión a la media ya no se aplica y el robot, si no se desconecta, realizaría ruinosas operaciones con pérdidas. Para dirigir el robot, el trader de arbitraje estadístico aprovecha la presencia de humanos en el resto de la sala. Por ejemplo, el trader obtiene pistas decisivas sobre qué empresas están a punto de fusionarse cuando oye por casualidad conversaciones en la mesa adyacente, la de arbitraje de fusiones. De modo semejante, el supervisor humano del robot utiliza la sala para descubrir si los datos que llegan al robot están experimentando algún retraso (y, por lo tanto, creando una peligrosa tergiversación de los precios reales). Ello se hace no sólo prestando atención a los indicadores incorporados al robot, sino también a las quejas y al posible pánico entre los técnicos informáticos que se sientan cerca. Si el arbitrador estadístico oye gritos y exclamaciones, ello quiere decir que hay problemas técnicos, aunque los indicadores del monitor indiquen «OK».

La sala de contratación de International Securities, como las del WTC que desaparecieron en el ataque, constituían un conjunto único de elementos sociales, espaciales y técnicos que es necesario entender para apreciar lo que significaban las finanzas en el Bajo Manhattan. En este exclusivo mundo, las relaciones sociales tienen mucho valor: el proceso interpretativo que tenía lugar en él recurría a relaciones sociales no jerárquicas, relaciones laterales basadas en la confianza. El espacio también era importante: la sala y su configuración espacial basada en escritorios promovían la comunicación y distribuían el conocimiento a través de los equipos. Y la tecnología también importaba. La sala contaba con tecnologías de negocio altamente automatizadas, tales como robots y máquinas comerciales, y estas tecnologías se aprovechaban de la comunicación constante entre los traders, permitida por la localización en un espacio común.

\section{REUBICACIÓN: LAS REDES SOCIOTÉCNICAS, REVELADAS}

El once de septiembre, una ensordecedora explosión detuvo en seco el trabajo de los traders de International Securities. El impacto del primer avión de los terroristas precipitó a los traders hacia las ventanas del este de la sala. Allí vieron el edificio de enfrente, el World Trade Center, arder en llamas. El impacto del segundo avión provocó el terror en la sala y 
una huida tumultuosa hacia el río Hudson. Cuando se desplomaron las torres, los traders estaban ya alejándose de Manhattan, en el ferry de Nueva Jersey. Afortunadamente, ninguno de los empleados de la sala de renta variable de International Securities sufrió daño físico alguno. Su sede, sin embargo, quedó seriamente afectada, y con su sala de operaciones inaccesible. El Trade Center se había derrumbado por completo. Sus ventanas se habían hecho añicos con la explosión y quedaron perforadas por los escombros caídos de las torres. Toneladas de polvo, ceniza y suciedad, posiblemente con asbesto y sustancias químicas tóxicas, habían entrado en la sala, penetrando en los ordenadores, atascando sus ventiladores, sobrecalentándolos y haciéndolos inservibles. Los datos que contenían estaban perdidos. El edificio fue calificado como estructuralmente inseguro y se prohibió el acceso al mismo durante meses. En consecuencia, la activa sala de operaciones que en otros momentos había sostenido un trabajo de interpretación devino una cueva negra, sin electricidad, sin conectividad y sin ninguna garantía de seguridad.

En una reunión de urgencia en la noche del once de septiembre, el equipo gerente de la sala de operaciones de International Securities resolvió que la recuperación sería larga y dura, y que volver a operar llevaría de tres semanas a tres meses. A diferencia de algunos otros bancos, International Securities carecía de una oficina de emergencia. El banco contaba con otras instalaciones, como una oficina administrativa (backoffice) en la periferia de Nueva Jersey, pero el único recurso con el que podían contar allí los traders era espacio libre en un sótano donde la empresa almacenaba miniordenadores para procesar la nómina. El sótano no tenía terminales Bloomberg, ni mesas, ni conectividad.

Con todo, apenas seis días después del once de septiembre, cuando la Bolsa de Nueva York reabrió sus puertas el 17 de septiembre, los traders de International Securities estaban de nuevo operando. Tuvimos el privilegio de presenciar este logro. Varios días después del ataque, enviamos al banco un correo electrónico interesándonos por el bienestar de los empleados. Para alivio nuestro, supimos que nadie había resultado herido. Para sorpresa nuestra, la respuesta al correo que enviamos incluía una invitación —más bien, una solicitud-para que nos acercáramos a Nueva Jersey a presenciar el proceso de recuperación. «Es caótico», escribió el gerente de la sala de operaciones, «pero también muy inspirador». Nuestra presencia sería «un recuerdo de tiempos normales». Como etnógrafos, nos sentimos enormemente honrados de ser bienvenidos para documentar este extraordinario esfuerzo.

De este modo, el 19 de septiembre de 2001 volvimos a nuestro papel de observadores entre los traders, pero esta vez en una sala de operaciones improvisada en un sótano-almacén en Nueva Jersey. La sala estaba apenas a una hora de coche desde Manhattan. Pero, dada la falta de excitación y actividad de Wall Street, Manhattan quedaba realmente lejano. 
Ubicado en un parque empresarial suburbano, el edificio estaba rodeado de oficinas de baja altura de empresas de manufactura como Colgate o AT\&T. En la esquina, un cartel en una granja anunciaba: «se vende heno». Los alrededores ofrecían una continua sucesión de centros comerciales, Wal-Marts y Dunkin Donuts, por los que uno podía dar vueltas durante una hora sin poder encontrar un café exprés. Lo que había sido el backoffice u oficina administrativa de International Securities se había convertido, en efecto, también en su frontoffice o sala de operaciones. Nuestros traders eran traders de Wall Street... en Nueva Jersey.

La sala estaba ubicada en el sótano del edificio. Para llegar a él tuvimos que pasar por varias filas de cubículos y alfombra beige de corte corporativo. Y, tras los cubículos, una inesperada escena: la sala de operaciones. Un gran espacio de planta abierta, completo con traders, escritorios, ordenadores, pantallas de televisión y clásicos relojes de Wall Street con varias franjas horarias. La sala tenía un aspecto provisional: no tenía ventanas, el techo era bajo y las paredes estaban pintadas de amarillo industrial, más adecuado para un almacén que para una sala de operaciones. Y, en efecto, una semana antes de nuestra visita el lugar todavía se usaba para almacenar los miniordenadores y las cintas utilizadas por el centro de procesamiento de datos del banco. Los conductos de aire acondicionado a ras de suelo, diseñados para enfriar grandes ordenadores, todavía funcionaban el día de nuestra visita, enfriando nuestras pantorrillas de zapato para arriba. Dentro de la sala, los técnicos de informática se afanaban constantemente entre cables de repuesto, teclados y ratones mezclados con latas vacías de Diet Pepsi.

Nuestros traders no eran sólo arbitradores provisionales: eran supervivientes. «No tengo que decirles lo cerca que estuvimos», nos dijo uno de ellos, refiriéndose a su apurada huida del World Financial Center. Una gran bandera americana colgaba de la mitad de la pared, y docenas de otras más pequeñas colgaban de las pantallas de otros tantos traders, como flores en un campo en primavera. De los tres grandes televisores en la sala, tradicionalmente sintonizados al canal financiero CNBC para recibir noticias del mercado, uno estaba sintonizado a la CNN para recibir noticias de la inminente guerra en Afganistán. La vestimenta había pasado del elegante-informal de Wall Street a vaqueros y botas. La sala era ruidosa, pero el ruido, como dijo un trader, era «un maravilloso ruido de vida».

Nuestros traders estaban, indudablemente, en un sótano-almacén de Nueva Jersey. Pero un letrero llamativo en la pared daba coordenadas diferentes: «Piso 20, Renta Variable». En otras esquinas de la sala podían leerse indicaciones similares: «Piso 21, Renta Fija» y «Piso 19, Gestión de Riesgos». Nuestros traders todavía estaban entre los pisos 19 y 21, pero ahora el orden era horizontal en lugar de vertical. Además, dentro de las restricciones de aquellas habitaciones, los traders habían organizado sus mesas para reproducir el or- 
den de la sala del Financial Center. Por ejemplo, todos los traders de la mesa de operaciones de clientes se mantenían juntos. En la sala del Financial Center disfrutaban de una amplia mesa entre la de bonos y la de situaciones especiales. En Nueva Jersey acampaban en una mesa parcialmente ocupada por dos fotocopiadoras y tres faxes, en lo que solía ser la esquina del fax del centro de datos. Acampaban, pero juntos.

Los escritorios también conservaban sus ubicaciones relativas, reconstruyendo el orden mental de la sala de operaciones del Financial Center. Una vez los gerentes de la mesa de órdenes de clientes y la de situaciones especiales se encontraron sentándose nuevamente frente a frente, volvieron a su vieja rutina de contrastar percepciones entre sí, investigando las creencias de los demás y diseñando juntos nuevas operaciones de arbitraje. «Todos parecen estar pensando con mi cabeza», exclamó uno de ellos, agotado, en un momento del día, dejando manifiesto que el proceso de pensamiento distribuido había vuelto a la sala.

Los traders podían replicar la distribución de la planta de la sala de operaciones del Financial Center, pero no la tecnología. No era posible recibir datos desde el NYSE. «Trade Manager v.1.4a», la plataforma de hardware y software que registraba y procesaba las operaciones (el «motor financiero»), no funcionaba. Las centralitas telefónicas usadas habitualmente por los traders, con 20 líneas cada una, tampoco estaban disponibles, de forma que los traders tenían que trabajar con teléfonos de una sola línea (que, sin embargo, colgaban con la misma fuerza de siempre). En lugar de sofisticados terminales de Sun Microsystems, trabajaban con clónicos Pentium II y ordenadores portátiles. Algunos los habían traído de casa, otros habían sido rescatados del centro de datos, y otros habían sido adquiridos apresuradamente en los días siguientes al ataque. Pero, en lugar de tener un ancho de banda virtualmente ilimitado, ahora tenían que adaptarse a conexiones en red limitadas que impedían la compra-venta simultánea por parte de todas las mesas de la sala.

La respuesta de los traders al once de septiembre presenta importantes conclusiones para la perspectiva sociotécnica de las organizaciones. En los apartados anteriores hemos sostenido que los arbitradores asocian títulos financieros mediante la asociación de personas, artefactos e ideas en el mismo lugar. Conceptualmente, es tentador dividir esta red sociotécnica en seres humanos y máquinas: personas que piensan y hablan contra máquinas que obedecen órdenes programadas con anterioridad. Pero tal separación es errónea. «La tecnología», escribe Bruno Latour (1991, p. 1), «es la sociedad hecha duradera». Así, el ataque plantea las siguientes preguntas: ¿qué sucede cuando la tecnología deja de funcionar, cuando traders que estaban acostumbrados a 20 líneas de teléfono para cada uno tienen que compartir teléfono, cuando traders cuya estrategia se basa en la velocidad y el volumen deben repentinamente operar con un ancho de banda mínimo? La quiebra de la 
tecnología financiera en International Securities abrió un resquicio en el que observar la red sociotécnica, una red que operaba perfecta e invisiblemente en la sala de operaciones del Financial Center. La ruptura de la tecnología supuso la sociedad hecha visible.

La ruptura de la tecnología reveló también la forma en que personas y artefactos estaban inextricablemente unidos. Por ejemplo, al escuchar el proceso por el cual el banco estableció una conexión con el NYSE, observamos que el jefe de informática de International Securities utilizaba «conexión» y «relación» de forma indistinta. En algunas ocasiones se referiría a «Mike» y en otras a «la conexión ISDN», queriendo decir la misma cosa. El primer intento para conectar la sala de operaciones con el NYSE se realizó a través de redes de comunicación electrónicas (electronic communication networks, o ECNs), pero la conexión se interrumpía a cada minuto, lo cual era muy problemático para los traders porque no podían saber exactamente cuál era su exposición al riesgo. Al final, el banco logró conectar con el NYSE sólo a través de una ECN que llevó a sus técnicos a la sala de operaciones. Y, a su vez, la única razón por la que la ECN invirtió sus recursos en ello (humanos, financieros, etc.) fue la relación de negocios que la unía con International Securities, y porque estaba interesada en que el banco operara a través de su sistema y le aportara volumen y liquidez. De ahí el estrecho vínculo entre relaciones sociales y técnicas. Como explicó el jefe de tecnología, «una vez que establecemos una relación con alguien, es muy fácil progresar» hacia una conexión. Una implicación de ello es que las empresas con amplias redes sociales deberían recuperarse más fácilmente de problemas con su tecnología.

Como sociedad hecha duradera, la tecnología de International Securities también reflejaba el entorno regulador en el que se desarrolló. En el proceso de reconexión de la sala de operaciones en Nueva Jersey con el NYSE, nuestros traders experimentaron grandes dificultades para encontrar módems adecuados para sus máquinas. La razón era que, en el pasado, la regulación había limitado a los bancos a una velocidad de 9,6 K de transmisión para las conexiones con el NYSE, a fin de evitar inútiles inversiones en tecnología a modo de carrera armamentística. Así, la tecnología es también regulación hecha duradera. Sin módems especialmente configurados, los traders de Nueva Jersey no podían enviar y recibir datos a y del NYSE. Pero en septiembre de 2001 los antiguos módems de 9,6 K no podían obtenerse a través de los canales comerciales habituales. Como explicó el jefe de tecnología, el banco trató incluso de rescatarlos del Financial Center para poder seguir operando:

«Los módems estaban en los viejos ordenadores Unix, y no pudimos encontrar módems nuevos para nuestros ordenadores. Así que tuve que volver al World Financial Center para desmontar los ordenadores, subiendo veinte pisos con un traje químico y alumbrándome con una linterna porque no había electricidad». 
Una red sociotécnica es mucho más compleja que la simple suma de las relaciones sociales y técnicas en la organización. La ruptura de las relaciones técnicas, por ejemplo, no puede repararse automáticamente por nuevas relaciones sociales. Esto se ve claramente en el cartel de «Piso 20, Renta Variable» colocado en la pared y su insistencia implícita en reproducir la antigua estructura de las mesas. El cartel no sólo recordaba a los traders que la sala de operaciones en renta variable estaba situada entre las de gestión de riesgos y renta fija, sino también hacía volver a los empleados a sus puestos de trabajo como traders. Mediante la reconfiguración de la red sociotécnica que había desaparecido, reducía la incertidumbre fundamental a la que se enfrentaban los traders. Según Callon, una red sociotécnica

«... no conecta identidades que ya están allí, sino una red que configura ontologías. Los agentes, sus dimensiones y lo que son y hacen, todo depende de la morfología de las relaciones en que están implicados» (Callon, 1998, p. 15).

Después del ataque, los traders se preguntaban si su empresa continuaría existiendo, si la sala de operaciones operaría de nuevo; se preguntaban lo que ellos harían e incluso lo que serían. El sótano devolvió a aquellos supervivientes la condición de traders. A la pregunta «¿quién soy yo?», los ordenadores, los escritorios y la planta abierta respondieron «un trader». A la pregunta «¿qué tengo que hacer?», el cartel de «Piso 20» contestó «lo mismo que harías en la sala de operaciones del Financial Center».

El carácter ontológico de la red sociotécnica también se manifestaba en el discurso de los traders. Los encontramos ocupados en un debate sobre el significado de qué constituyen "datos reales». El problema al que se enfrentaban los traders era que las conexiones con datos directos propietarios que unían a International Securities con el NYSE llegaban directamente al Financial Center y, por lo tanto, no podían utilizarse en Nueva Jersey. Los traders tenían que contar a cambio con datos de Bloomberg L.P. «Pero», lamentaban los traders, «los datos de Bloomberg no son reales». Tenían pequeños e imprevistos retrasos que los hacían inapropiados para algunas estrategias comerciales como el arbitraje de índices. Así, los traders tenían que efectuar operaciones reales con datos que ellos no consideraban reales.

Los traders también se ocuparon en reparar activamente su posición como pilares económicos de la organización. Ante las tecnologías dañadas y las herramientas desaparecidas, volvieron a combinar viejas y nuevas herramientas a fin de poder operar de nuevo. En la mesa de operaciones de clientes, por ejemplo, los jóvenes traders de menor rango realizaban manualmente operaciones que previamente se habían automatizado por medio del motor financiero, tales como inscribir operaciones, registrarlas, terminarlas, etc., usando tecnología financiera de cinco años atrás: papel y lápiz. A falta de sillas, los traders jóvenes permanecían de pie, dispuestos a ayudar, detrás de aquellos con mayor rango que conta- 
ban con silla y ordenador. Cuando, en medio de una conversación telefónica, uno de los traders necesitó registrar una transacción, al grito de «idadme un ticket, que alguien me dé un ticket!», tres jóvenes traders de a pie se apresuraron a ofrecer tickets, papel y lo que pudiera necesitar. Otro trader joven fue enviado a «ayudar con los tickets» y a «asistir a otros» en una mesa distinta. Pero la orden le fue dada con la sensibilidad característica de International Securities: el trader de mayor rango le dio las indicaciones añadiendo «ah, y esto no es definitivo, por cierto». La inscripción manual de operaciones era tan insólita para los traders más jóvenes — tan radical era el bricolaje que implicaba-que algunos de ellos ni siquiera sabían cómo hacerlo.

De modo semejante, los traders de arbitraje estadístico compensaban la falta de datos directos del NYSE a base de transformarse. Pasaron de supervisores de sus robots financieros a participantes activos en el mecanismo de precios. «Bienvenidos al país del cortar y pegar», nos dijo un arbitrador estadístico a modo de saludo, refiriéndose despectivamente a su incesante actividad de transporte de órdenes de compra-venta de su programa de correo electrónico al motor financiero, haciendo clic y arrastrando con el ratón. Procedía así porque la falta de información sobre precios en el sistema Unix del motor financiero le obligaba a conectar manualmente un interfaz con el otro. «Por eso», explicaba el trader, «no tengo tiempo para hacer otras tareas» como supervisar el mercado y la velocidad de los alimentadores de precios, su trabajo típico. Así, el trader de arbitraje estadístico había resuelto operar sin reparar el motor financiero, haciéndolo manualmente.

En International Securities, la escasa conectividad originó una situación en la que no todos los traders tenían suficiente ancho de banda para operar simultáneamente. Por ejemplo, cuando la mesa de arbitraje de índices estaba activa, las otras no podían comprar ni vender. Aunque ello hacía que el bonus de los arbitradores de índices fuera a costa de los de otros equipos, el resto de los traders no dejaron que la situación terminara en conflicto. El episodio es un ejemplo de otro rasgo del bricolaje igualmente importante: la tolerancia ante una situación que no es la ideal.

¿Cuál es la lección que se extrae de esta provisional sala de operaciones para la organización de la respuesta a una crisis? La experiencia de estos traders sugiere que responder acertadamente requiere una combinación de previsión y de improvisación. El banco tenía un espacio, pero distaba de ser una réplica perfecta de la sala de operaciones del Financial Center. Con todo, los traders consiguieron estar operando allí desde el primer día. ¿Cómo? Recurriendo al bricolaje. El banco tenía un almacén con apenas unos cuantos metros cuadrados. En ese espacio, los traders vieron un recurso que utilizaron para organizar las mesas casi con la misma configuración que en su anterior sala de operaciones. Las herramientas que los traders tenían a su disposición eran rudimentarias en comparación con los 
instrumentos de precisión a los que estaban acostumbrados. En Nueva Jersey tan sólo tenían teléfonos con una sola línea, ordenadores portátiles, una conectividad reducida y terminales de una sola pantalla. Pero los hacían funcionar: lograron comunicarse con otros bancos, pasar órdenes y conectar con el NYSE. Como artistas del bricolaje, los traders no permitieron que la falta de condiciones perfectas les impidiera llevar a cabo las tareas. En lugar de esperar a que el motor financiero estuviera reparado o a que les entregaran los nuevos servidores, los traders recombinaron nuevas y viejas tecnologías rápidamente. Ajustaron sus ropas de artistas del bricolaje — vaqueros y botas- a su entusiasmo para resolver problemas. En este proceso, algunos se transformaron en oficinistas, otros en operadores manuales y otros en compañeros de piso de ancho de banda, compartiendo cable con el NYSE. Estos cambios de rol no les apartaron de su status como traders; de hecho, reafirmaron su status como tales. A veces, las cosas tienen que cambiar para seguir igual. Sus identidades como traders estaban grabadas en sus tarjetas profesionales. ¿Qué hacen los traders? Operan. Los traders encontraron maneras de operar reubicándose en las redes sociotécnicas dañadas por el ataque. La innovación no consiste en tener nuevos recursos para llevar a cabo nuevas tareas, sino en reconocer configuraciones que otros no verían como recursos. La respuesta se basa en este reconocimiento de los recursos.

\section{LA RECUPERACIÓN COMO INNOVACIÓN}

Después de nuestras observaciones en International Securities, tuvimos que preguntarnos si el nuestro era un caso típico o excepcional. ¿Qué había sucedido en otras empresas? ¿Cuál fue la experiencia de las empresas del Trade Center, aún más directamente afectadas por el ataque? Comenzamos a hablar sobre respuesta y recuperación con numerosas empresas del WTC. Conversamos con gerentes de empresas grandes, medianas y pequeñas, individualmente y en grupos. E hicimos esfuerzos extraordinarios para hablar con los responsables de tecnología, planificación de emergencias, preparación y gestión de continuidad. Como parte de este esfuerzo, el Center on Organizational Innovation de la Universidad de Columbia, junto con el Interactive Design Laboratory de Columbia, organizaron una mesa redonda el 5 de diciembre de 2001 con altos responsables de informática y comunicaciones de empresas clave del Trade Center, así como con empresas de consultoría en tecnología. Éstas incluían a Merrill Lynch, Cantor Fitzgerald, Deutsche Bank, Sun Microsystems, Guy Carpenter, Accenture y Fred Alger Associates. La mesa redonda no estaba abierta al público. Prometimos a los participantes que en este trabajo solamente informaríamos de sus comentarios, sin atribuirlos a personas o empresas concretas ${ }^{7}$.

7 Para más información sobre la mesa redonda con empresas del WTC afectadas por el once de septiembre, véase http://www.coi.columbia.edu/pdf/infrastructure_interface_program.pdf. 
¿Cuáles fueron sus comentarios? Nadie nos dijo: «David, fue la tecnología lo que nos salvó» o «Daniel, nuestro plan realmente funcionó». A pesar de ser directivos de informática, todos ellos señalaron las relaciones sociales como un rasgo clave de la respuesta organizativa. Por supuesto que hablaron de planes de emergencia y de tecnología. Nos dijeron, por ejemplo, que un aspecto clave de la recuperación fue que el Trade Center hubiera sufrido los efectos de una bomba con anterioridad, en 1993, y que su planificación y preparación subsiguiente marcaba una diferencia, o que las copias de seguridad en preparación para el virus del año 2000 habían demostrado ser muy importantes en la recuperación. Pero una buena planificación no fue suficiente para afrontar la incertidumbre creada por un desastre semejante. Según cinco de los ejecutivos que hablaron con nosotros:

«Sin el elemento humano de dedicación a la tarea y dedicación mutua, la preparación no habría servido de nada. El mejor plan no habría podido desarrollarse jamás».

«No podríamos haberlo hecho sin las tecnologías corporativas que teníamos, pero lo que nos sorprendió, en las primeras horas y días después del ataque, fue lo importantes que eran las tecnologías [localizadores, teléfonos celulares privados, Blackberries, Palm Pilots, etc.] en las que la empresa no había invertido».

«Un plan de negocio es una cosa, pero se necesita un plan de personas, y todo el mundo necesita una responsabilidad».

«Estoy seguro de que la preparación contribuyó... pero incluso donde no la había, la gente innovó precisamente en torno a ella».

«Si se está hablando sobre la medición de la preparación, la cuestión clave es cómo pudieron realmente las personas trabajar juntos y colaborar».

Podemos resumir nuestros hallazgos de la siguiente manera. El éxito en la recuperación fue resultado de una combinación de planificación y espontaneidad, de redundancia y autoorganización. Para estructurar estas abstracciones presentamos algunos relatos de recuperaciones de éxito en palabras de los participantes en el grupo de enfoque.

El primer relato recoge las impresiones de un ejecutivo de una gran empresa de renta variable del Trade Center que sufrió terribles pérdidas. La tarde del once de septiembre, los supervivientes se reunieron sabiendo que tenían que estar operando cuando el mercado de bonos abriera esa misma semana. La empresa había seguido todas las directrices de planificación de emergencias. Habían guardado sus datos en copias de seguridad $-\mathrm{y}$ no 
sólo una, sino dos copias - en lugares diferentes y fuera de la oficina: una al otro lado del río Hudson y otra al otro lado del Atlántico. Pero no podían entrar en el sistema. Como cuenta un ejecutivo:

«Teníamos cuarenta y siete horas para [prepararnos] para el trece de septiembre, cuando volvería a abrir el mercado de bonos, y nuestro departamento de tecnología tenía que invertir su tiempo en una sola tarea... Entrar en los sistemas, [descifrar] las contraseñas de los sistemas. Había muerto tanta gente, precisamente la que sabía cómo entrar y que conocían la copia de seguridad, la persona de emergencia, todos habían desaparecido. ¿Cómo entraron en aquellos sistemas? Nos sentamos alrededor en grupo, y hablamos de dónde iban de vacaciones, de cuáles eran los nombres de sus hijos, de sus esposas, de sus perros... sabes, cualquier cosa que pudiera imaginarse acerca de su vida personal. $Y$ el hecho de que supiéramos cosas sobre su vida personal para entrar en los sistemas para poder organizar la tecnología y hacerla funcionar antes de que abriera el mercado de bonos, creo [que] es probablemente la principal conexión entre tecnología, comunicación y personas».

Con un relato tan emocionalmente convincente, el investigador debe, en primer lugar, hacer una pausa y contemplar el sufrimiento de aquel momento, ejemplar en tantos aspectos. A las cuatro de la madrugada habían abierto los sistemas; pero las historias que contaban, debemos recordarlo, eran relatos de pérdida personal.

El relato es ejemplar, por supuesto, también en un aspecto analítico. Primero, es una emocionante ilustración de la percepción de que la crisis revela que cualquier tecnología es siempre socio-tecnología. Todos los sistemas técnicos de copias de seguridad estaban presentes, pero sin el conocimiento - conocimiento, en este caso, de las contraseñas del sistema- no se tenía tecnología ni información. El interfaz entre humanos y tecnología y datos era social (Kelly y Stark, 2002). Segundo, las habilidades necesarias para acceder al conocimiento codificado (literalmente codificado: a los códigos del sistema) no estaban a su vez codificadas. El caso ilustra el desafío planteado por Orlikowski (2002) a la distinción entre codificado y no codificado, y otras dicotomías relacionadas. Como razona ella, la puesta en práctica eficaz del conocimiento, o «conocimiento en práctica», requiere combinaciones de conocimiento explícito y tácito. Los códigos del sistema estaban accesibles a través de un conocimiento personal, no codificado, en interacción con algoritmos que traducían el conocimiento personal a contraseñas reales ${ }^{8}$.

\footnotetext{
8 Al descubrir las contraseñas, los técnicos teclearon el nombre del miembro de una familia para convertirlo en una notación numérica de los modelos de números y letras en un teclado numérico de teléfono, y viceversa para las fechas de nacimiento en letras, o multiplicando, sumando, invirtiendo o mezclando números y letras, y así sucesivamente. Es decir, en este caso, el interfaz era también socio-técnico.
} 
En tercer lugar, el relato revela qué forma organizativa mantiene tal conocimiento. Los empleados sabían los nombres de los cónyuges de sus colegas o dónde iban de vacaciones o sus películas o música favoritas; pero no porque la organización hubiera investigado formalmente y hubiera incorporado esta información en alguna base central de datos. La clave en este caso era lo bien que se conocían personalmente entre sí: detalles de la vida privada que eran estrictamente irrelevantes para su status como compañeros de trabajo. Dado nuestro trabajo de campo previo en la sala de operaciones, no nos sorprendimos de que los empleados compartieran tal conocimiento. Nuestras entrevistas indican que tales relaciones se extendían más allá de los equipos de trabajo inmediatos. Nuestros interlocutores enfatizaban repetidamente la importancia de la «formación cruzada» y de las «relaciones laterales más allá del departamento» como recursos en red en la crisis. En resumen, la gente se conocía mutuamente en múltiples registros dentro de equipos y a través de departamentos. Dentro de estas empresas no había una sola lógica, sino múltiples lógicas, en las que los actores eran expertos en códigos que cambiaban (White y Mische, 1998).

La capacidad de utilizar múltiples códigos, incluyendo no solamente el verbal, sino también el no verbal, fue decisiva para la recuperación, porque las empresas estaban trabajando con personal afectado por el miedo y el dolor:

«Esto no era un incendio en un edificio que acababa de destruir dos plantas... La mayoría perdió a gente que conocía. Estaban traumatizados, había temor a la guerra. Nadie sabía si el ataque continuaría al día siguiente. Tenía un tipo dando vueltas con una foto de su esposa y sus chicos en su bolsillo y la miraba cada dos minutos porque tenía miedo de no poder volver a su casa».

En estas circunstancias, como observó un directivo, «lo que marcó la diferencia en cada empresa que se recuperó con éxito fue cierto tipo de sensibilidad, mucho tacto, poca tecnología».

Los fuertes vínculos sociales en los equipos fueron particularmente importantes en las primeras horas después del ataque. Por ejemplo, saber dónde se encontraban los demás cuando las líneas telefónicas dejaron de funcionar:

«Te das cuenta de que el edificio se ha derrumbado. Hay un momento en el que crees realmente que eres la única persona que queda viva en la empresa y creo que desde el principio fue algo más instintivo que organizativo. Una hora y media después de que el primer avión chocara contra la torre, los cuatro compañeros restantes de mi equipo (yo tenía diez en el Trade Center) estaban en mi puerta. Habían 
venido desde el centro de la ciudad, desde el metro, el vestíbulo, desde donde quiera que estuvieran, y aparecieron en mi puerta por ninguna otra razón que el convencimiento de que teníamos que hacer algo».

De modo semejante, también fueron decisivos los límites organizativos difusos que llevaban a fuertes vínculos sociales con clientes, traders y asesores. Según otro ejecutivo del Trade Center:

«Los comerciales y proveedores en nuestras áreas de informática, telecomunicaciones y casi todas las áreas en general, estuvieron extraordinarios. Es habitual criticar a esta gente y hacer acerca de ellos blanco de las bromas mas duras. Pero en este caso fueron extremadamente generosos. No se imaginan cuánto confiábamos en las relaciones que teníamos con comerciales, asesores y clientes. Esta gente estaba dispuesta a hacer lo que fuera para volver a conectarnos, y si ello significaba trabajar las veinticuatro horas al día para que pudiéramos abrir el catorce [de septiembre], ellos lo harían. Esas relaciones no pueden reemplazarse con nada».

En resumen, al igual que los vínculos en las redes interorganizativas que promueven la innovación en las empresas de biotecnología (Powell, 1996), las relaciones sociales laterales fueron decisivas para la respuesta.

¿Cuál es la base organizativa de respuesta ante la incertidumbre? Podemos pensar, en primer lugar, en la respuesta ante las incertidumbres a las que se enfrentan las organizaciones como parte de sus operaciones «normales»: los negocios, las universidades, los museos, los hospitales y los organismos sin ánimo de lucro y del sector público se enfrentan con el imperativo de la innovación organizativa cuando se encuentran en condiciones de incertidumbre radical. Esa incertidumbre viene provocada por una extrema volatilidad de los mercados y un rápido cambio tecnológico. Para poder subsistir con esta incertidumbre, las organizaciones están aplanando sus jerarquías, distribuyendo sus fuentes de información, promoviendo estructuras de colaboración y aumentando la coordinación lateral para ganar en flexibilidad. Pero también podemos pensar en la respuesta como reacción a la incertidumbre radical provocada por una crisis como la que vivimos el once de septiembre. Nuestra investigación indica que los nuevos avances de la teoría organizativa tienen aplicabilidad al pensamiento sobre preparación en respuesta a las crisis.

A mediados del siglo xx existía un consenso general sobre los atributos ideales de la organización moderna: había una cadena de mando clara, con estrategias y decisiones adoptadas por la dirección organizativa; las instrucciones se difundían y la información se reunía arriba y abajo de la escala jerárquica de la autoridad; el diseño precedía a la ejecución, 
realizándose esta última con la precisión de una gestión del tiempo propia de una máquina organizativa taylorista. A finales del siglo, los principales preceptos del modelo organizativo ideal se reescribieron de manera fundamental. La primacía de las relaciones de dependencia jerárquica dentro de la organización y las relaciones de independencia de mercado entre las organizaciones pasó a ser secundaria respecto de las relaciones de interdependencia entre las redes de organizaciones y entre las unidades dentro de las organizaciones (véanse los ensayos de Powell, Stark y Westney en DiMaggio, 2001).

La visión tradicional sobre la capacidad de preparación tiene mucho en común con la visión de mediados del siglo xx sobre la organización eficaz: establecer un plan para la gestión de la emergencia con una cadena de mando clara. En el centro de tal pensamiento se encuentra el concepto de redundancia, es decir, tener sistemas de seguridad que repliquen las funciones cruciales en caso de avería: más de un sistema telefónico, un sistema informático, una sala de operaciones, etc. (Kelly y Stark, 2002). El once de septiembre demostró la importancia de tales sistemas redundantes. Tener una sala de operaciones de seguridad, como hicieron algunos bancos de inversión como consecuencia de los planes de emergencia instrumentados después del ataque al Trade Center en 1993, fue la clave para garantizar la continuidad de las operaciones de negocio. Este concepto tiene mucho mérito: ninguna sala de operaciones puede sobrevivir, por ejemplo, sin una copia de seguridad de sus datos. Pero los límites de la planificación para las crisis se parecen a los límites de la planificación cuando los horizontes estratégicos son complejos (Lane y Maxfield, 1996): cuando el futuro es radicalmente incierto ${ }^{9}$, no se puede conocer por adelantado qué recursos se necesitarán, ni siquiera saber de antemano lo que podría ser un recurso.

Por lo tanto, además de la redundancia replicativa, nuestra investigación sugiere también la importancia de la redundancia generativa en la respuesta a las crisis. Esta redundancia se diferencia de aquélla que solamente duplica el mismo tipo de recurso (Grabher, 1994; Grabher y Stark, 1997). La redundancia generativa es una «redundancia» diferente. Y es por esta razón que puede ser generativa. En situaciones de incertidumbre radical, la diversidad de relaciones y de medios aumenta la probabilidad de que la interacción produzca soluciones impredecibles por medio de «abrasión creativa» y «fricción generativa» (Brown y Duguid, 1998; Girard y Stark, 2002). Las relaciones laterales que trascienden las estructuras verticales oficiales son redundantes pero no simplemente replicativas; conocer a sus compañeros de trabajo, el número de teléfono de su casa u otras maneras de ponerse en contacto con ellos es una redundancia no replicativa; y las organizaciones que admiten

9 Como observó uno de nuestros informantes, «ya sabes aquel comentario de Tolstoi que es algo así como "todas las familias felices son igual de felices, pero las familias infelices son excepcionalmente tristes". Lo mismo pasa con nosotros. Un día normal es como cualquier otro, pero toda crisis realmente grande es extraordinaria. He aquí por qué no se puede planificar exactamente lo que hacer ante una crisis». 
más de una manera de hacer las cosas están similarmente dispuestas a sacrificar alguna eficiencia asignativa a corto plazo en interés de una adaptabilidad dinámica. Estas redundancias facilitaron la autogestión cuando los nodos de la jerarquía corporativa fueron dañados o destruidos, y permitieron la redefinición flexible de roles y recursos en un momento de crisis.

Esta discusión es pertinente no sólo por cómo pensamos acerca del estado de preparación, sino también por cómo podría medirse el grado de preparación en un contexto de valoración financiera de empresas. Un ejecutivo de la mesa redonda observó que los acreedores y los inversores prestarían una atención minuciosa al estado de preparación como factor en las valoraciones financieras de las empresas después del once de septiembre:

«Lo que más hemos aprendido en cuanto a estrategia y previsión de futuro es que ahora las empresas que miran al futuro van a ser valoradas por algo que yo llamo estado de preparación. Creo que ello se va a convertir en una parte integral de la forma en que inversores, empleados, acreedores, todo el mundo que examina el valor de una empresa. Se volverá análogo y tal vez incluso parte de su calificación de crédito, y estos temas de estado de preparación se reducen a algunos de los aspectos más triviales... como procesamiento del correo, viajes en avión, seguridad en el trabajo, seguridad de los datos e infraestructura de los datos, identificación personal y responsabilidad personal, e incluso la elección de nuevos clientes y fuentes de financiación».

En la visión convencional existe una relación inversa entre estado de preparación y competitividad. La redundancia replicativa es una práctica empresarial necesaria, pero es un coste neto que no contribuye a la competitividad organizativa. Esta visión del estado de preparación da ventaja a las empresas más grandes y burocráticas. En contraste, la redundancia generativa podría contribuir al estado de preparación a la vez que aumenta la efectividad y competitividad. Las estructuras heterárquicas que contribuyen a la capacidad de respuesta en épocas de crisis pueden facilitar la creatividad de la organización en sus respuestas al entorno de modo más general.

\section{EL CAMBIO DE LA GEOGRAFÍA URBANA DE LAS FINANZAS}

En abril de 2002, los traders de International Securities regresaron al World Financial Center, al igual que otras empresas como Merrill Lynch, Commerzbank y American Express, dando esperanzas de futuro del Bajo Manhattan como distrito financiero. Pero las opciones adoptadas por otras empresas afectadas por el once de septiembre sugieren más cautela que opti- 
mismo. A pesar del programa de incentivos económicos dirigido por la ciudad y el Estado de Nueva York para mantener a las empresas en el Bajo Manhattan, parece estar produciéndose un éxodo, con empresas como Lehman Brothers, Aon, Pillsbury Winthrop, Dresdner Kleinwort Wasserstein y ABN Amro abandonando el área en dirección a lugares más caros en el Medio Manhattan o a oficinas más alejadas en Brooklyn y Jersey City (Bagli, 2002). Durante varias décadas, el distrito ha estado perdiendo una pugna contra el Medio Manhattan como lugar elegido por las empresas financieras, y la reubicación forzada ha acelerado esa tendencia. El debate sobre el desarrollo del próximo Trade Center (Sorkin y Zukin, 2002) debe considerar estas tendencias históricas, así como tener en cuenta cambios tecnológicos que, según algunos autores, eliminan la necesidad de un distrito financiero ${ }^{10}$. Nuestra investigación sobre el comercio antes del once de septiembre y sobre la dinámica de la recuperación después de aquella fecha tiene que ver directamente con este debate.

Durante las últimas décadas del siglo xx, Wall Street ha experimentado una verdadera revolución cuantitativa, basada en tres patas: conectividad a redes de alta velocidad, informatización de alta potencia y desarrollo de las finanzas matemáticas (Dunbar, 2000; MacKenzie y Millo, 2003; Knorr Cetina y Bruegger, 2002). Su efecto global sobre la elección de localización de las empresas financieras es incierto, porque cada una de las patas ejerce una fuerza a favor y en contra de la proximidad entre empresas.

Consideremos en primer lugar el ascenso de los mercados electrónicos. Aquellos que apoyan una visión utópica de un sector financiero virtual, desubicado y perfectamente competitivo, señalan el éxito del NASDAQ, el primer mercado puramente electrónico del mundo, y a sus imitadores en otros países ${ }^{11}$, como prueba de que la conectividad en red reduce la importancia de la proximidad física. Según The Economist.

«El Bajo Manhattan... puede ser el mayor mercado electrónico del mundo. En los días en que las cámaras de los bancos estaban repletas de bonos al portador y títulos de acciones transferidos por "corredores" después de efectuadas las transacciones, las empresas bursátiles tenían buenas razones para agruparse en una misma zona. Sin embargo, la proximidad es de poca o ninguna ayuda a la hora de efectuar las operaciones» (Anónimo, 2001, pp. 68-69).

\footnotetext{
10 Para perspectivas generales sobre la geografía de las finanzas, véanse los artículos de Corbridge y Thrift (1994), Leyshon y Thrift (1997), Martin (1999) y Clark et al. (2000).

11 EI DTB de Frankfurt y el MATIF de París (ambos fusionados en Eurex el año 2000), el LIFFE de Londres y las Bolsas de Estocolmo y Madrid ya han adoptado forma electrónica. Otros mercados, como el reciente ECN convertido en mercado, Island Futures Exchange LLC, comenzaron de forma electrónica. Y otros, significativamente el Chicago Mercantile Exchange, han desarrollado un sistema dual físico-electrónico, manteniendo sus parqués y desarrollando un sistema electrónico, Globex, que representa más un complemento que una amenaza (Muniesa, 2000; Milo, 2001; Scott y Barrett, 2002; Zaloom, 2002).
} 
Efectivamente, la disponibilidad instantánea de datos sobre precios, noticias del mercado y cualquier otro tipo de información procedente de Internet, de la televisión financiera y de otras fuentes es en parte lo que permitió prosperar a los mayores fondos de inversión mobiliaria de Norteamérica pese a estar ubicados lejos de Wall Street. Fondos como Fidelity, Janus o Vanguard están tan lejos del Bajo Manhattan como lo están sus respectivas sedes, en Boston, Denver y Filadelfia.

Pero algunos aspectos de la conectividad en red aumentan la importancia de la proximidad entre empresas. Cuando los mercados electrónicos ponen la información instantáneamente al alcance de todos, las diversas interpretaciones, impresiones y percepciones de los demás se convierten en la fuente clave de ventaja competitiva (Amin y Thrift, 1992; Thrift, 1994, 2000; véase también Grabher, 2002a, b). Esto supone dar un valor a la proximidad inmediata al nivel de lo microlocal: las operaciones de arbitraje rentables, como hemos visto en nuestra investigación en International Securities, son fundamentalmente ejemplos de innovación, y la innovación tiene lugar dentro de una íntima proximidad tal como un pasillo o una mesa compartidos (Allen, 1977; Kraut et al., 2002; Olson et al., 2002). El modo deliberado en el que los arbitradores de International Securities intentaron reconstruir el diseño de la sala de operaciones en Nueva Jersey revelaba lo extremadamente conscientes de estas dinámicas que eran los traders.

A nivel más agregado del distrito, nuestra investigación en International Securities ha demostrado que la proximidad a otras empresas financieras es decisiva para algunas estrategias financieras, tales como el arbitraje de fusiones y de bonos convertibles. En el caso del arbitraje de fusiones, por ejemplo, los traders apuestan sobre la probabilidad de una fusión. Como parte de su estrategia, tienen que determinar el compromiso de dos empresas para fusionarse, y al hacerlo así se encuentran con que es decisivo asistir a las presentaciones de las empresas. Como nos dijeron los traders, no basta sólo con escuchar la reproducción de la reunión en Internet: es necesario estar allí, ver las caras del público cuando las empresas hacen sus afirmaciones atrevidas, topar con antiguos colegas en los pasillos y comer con las personas involucradas. Hay ventajas adicionales en estar cerca de otros traders que ejercen las mismas prácticas: el descubrimiento de nuevas técnicas de negocio, dinámicas de contratación en el mercado de trabajo, etc. (Kim, 1991; Porteous, 1999).

Esta búsqueda de interpretaciones siempre más matizadas explica por qué empresas como Chase y Citigroup han desplazado sus operaciones bursátiles de alto valor añadido al Medio Manhattan a pesar de los mayores costes de alquiler con respecto del Bajo Manhattan (Berman, 2002). Puede decirse lo mismo del reciente traslado de Lehman Brothers a Times Square después del once de septiembre (una ubicación que, en el mejor de los casos, no es más segura que el Bajo Manhattan) o del planificado traslado de Cantor 
Fitzgerald a un área cercana a Union Square. Además, la evidencia de los modelos de reubicación de las empresas desplazadas por los acontecimientos del once de septiembre sugiere que la mayoría de empresas del WTC que han sobrevivido se han trasladado al Medio Manhattan: de una muestra de 500 sobre un total de 700 empresas del WTC, un equipo de periodistas de investigación del New York Times descubrió que 39 habían fracasado, 50 operaban desde casa y unas 270 se habían reubicado en direcciones de Manhattan por encima de Chambers Street; efectivamente, en el Medio Manhattan (Flynn et al., 2002). El Medio Manhattan no es sólo atractivo para los bancos por su red de transportes: mientras que los servicios de alojamiento y comodidad del área atraen a la gente de negocios que viaja y a las corporaciones no financieras, el distrito atraerá a las empresas de Wall Street, siguiendo una tradición no muy antigua en la que las empresas de Wall Street siguen a los dueños del capital.

De la misma manera, el ascenso de los mercados electrónicos, la difusión de la informática de bajo coste y el desarrollo de las finanzas matemáticas tienen efectos duales sobre la elección de ubicación por parte de las empresas de Wall Street. Tomemos el ascenso y difusión de las fórmulas matemáticas ganadoras del premio Nobel. Al codificar el conocimiento de cómo combinar valores en operaciones rentables en fórmulas matemáticas, las empresas multiplican sus posibilidades de beneficiarse del conocimiento que estas últimas contienen. Pero las posibilidades de imitación por parte de competidores se multiplican igualmente (Kogut y Zander, 1992). La presión resultante para proteger este volátil conocimiento llevó a Long Term Capital Management (LTCM), el fondo de arbitraje más grande y sofisticado de Norteamérica, a mudarse de Manhattan a Greenwich, Connecticut, con la esperanza de minimizar el contacto de sus traders con los rivales y evitar así la amenaza de imitación (para un relato de la caída de LTCM, véase MacKenzie, manuscrito no publicado).

El auge de las fórmulas financieras, sin embargo, ha tenido también el efecto contrario, contribuyendo a que las empresas se ubicaran cerca entre sí. En nuestra investigación en International Securities descubrimos que la empresa ha vuelto a enfocar sus estrategias operativas hacia negocios complejos y ambiguos que no pueden modelizarse fácilmente y reducirse a una fórmula. En lugar de ello, la empresa se apoya en el conocimiento tácito de los diferentes mercados y productos financieros sintéticos de sus diferentes mesas, y cuenta con la interacción entre mesas para reunir las diferentes piezas del rompecabezas. Esta estrategia actúa como una fuerza centrípeta cuando las mesas de International Securities buscan activamente la proximidad entre sí y con la comunidad de arbitraje de Wall Street.

La difusión de la informática de bajo coste tiene un semejante efecto doble de acercar y alejar a las empresas entre sí. Cuando la posibilidad de hacer transacciones a alta veloci- 
dad se pone al alcance de cualquier empresa financiera, la ventaja temporal que ésta confiere se ve erosionada. La respuesta de empresas como International Securities ha sido llevar a su personal de tecnología al núcleo de su práctica, contratando técnicos de sistemas con una formación transversal en finanzas para racionalizar sus motores financieros (contabilidad automatizada y conciliación de operaciones) o para ampliar el ancho de banda de las conexiones con el mercado, de modo que los robots financieros puedan comprar y vender a velocidades cada vez mayores. En International Securities, el personal de operaciones básicas se sienta en la misma sala que los traders con ingresos más elevados, apenas a una de distancia.

Por otra parte, la reducción en el coste de la informática ha hecho que los tradicionales departamentos de operaciones sean en menor grado fuente de ventaja comparativa, propensa a ser externalizada o reubicada en la periferia de la empresa. Es decir, mientras que el personal de tecnología está siendo llevado al núcleo de la sala de operaciones, el hardware está siendo reubicado en sitios alejados del mismo. Ésta es la fuerza que hay detrás del reciente desarrollo del puerto de Nueva Jersey. En los últimos quince años, los habitantes de Manhattan han sido testigos de la sorprendente transformación de ese área, pasando de un vecindario oficialmente declarado arruinado a un atractivo (casi sofisticado) espacio de edificios de oficinas con conectividad de alta velocidad. Son los puestos de avanzada de las divisiones operativas de los bancos de inversión como JP Morgan Chase. De modo semejante, Citigroup envió su división de operaciones fuera de Manhattan, a Queens, en busca de costes inmobiliarios más bajos y acceso más rápido para los empleados con sueldos medios. El traslado estuvo motivado por la reducción de costes y la comodidad. Esto queda claro al ver el mediocre edificio de Citigroup, un edificio de oficinas que sobresale casi completamente en un vecindario característico por sus modestas casas de baja altura, restaurantes tradicionales y negocios familiares.

Nuestra investigación sugiere, por tanto, que no se puede leer la futura geografía urbana de las finanzas directamente a partir de las tecnologías de compra-venta. La revolución cuantitativa —el ascenso de los mercados electrónicos, las fórmulas matemáticas y la informática de bajo coste- está cambiando activamente la forma de la geografía de Wall Street, pero de maneras complejas y mutidireccionales. La proximidad se ha convertido en decisiva para algunas empresas y obsoleta para otras, una fuente de beneficios para algunos departamentos y una amenaza a la existencia de otros. Pero, para decirlo con claridad, las aglomeraciones espaciales que resultan no estarán necesariamente en el Bajo Manhattan.

En el debate público sobre las finanzas y el Bajo Manhattan, muchos académicos, responsables políticos e incluso gente de negocios identifican a Wall Street con el NYSE. Esta 
identificación habría sido correcta para la mayor parte del siglo xx: las salas de operaciones comenzaron como apéndices del NYSE que los bancos de inversión construyeron dentro de sus rascacielos corporativos a fin de hacerse un nombre y procesar mejor la información que obtenían de él. Hoy en día, sin embargo, la ecuación que identifica a Wall Street con el NYSE está obsoleta. Nuestra investigación indica que el lugar real de las finanzas modernas no es la Bolsa, sino las salas de operaciones. En consecuencia, tenemos que abandonar las visiones de las finanzas en el Bajo Manhattan que tenían una forma radial o monocéntrica urbana —el NYSE rodeado por salas de operaciones - y aceptar en su lugar una comprensión multicéntrica de Wall Street. El Trade Center no era el back office del NYSE. Sus salas de operaciones eran algo más parecido a laboratorios científicos que réplicas de la Bolsa. Wall Street se comprende mejor como una red de salas de operaciones en la que cada nodo está sujeto al área por su proximidad a los demás, más que a la Bolsa ${ }^{12}$.

Aunque la proximidad a las otras empresas financieras es importante, esto no implica por sí mismo estar en el Bajo Manhattan. Creemos que las diversas funcionalidades que estaban concentradas en el distrito financiero tradicional quedarán separadas. En la pluralidad resultante de los microdistritos, Wall Street se convertirá en un concepto repartido, espacialmente distinto de la calle del Bajo Manhattan cuyo nombre proporciona la metonimia para el sector.

A las transformaciones en la geografía urbana de las finanzas antes citadas, el once de septiembre ha añadido un nuevo elemento: la seguridad. En el atentado con bombas del WTC en 1993 y la preparación para el virus del año 2000, las empresas financieras aprendieron la importancia de los servicios de seguridad y de la planificación de emergencias. La lección del once de septiembre, según los ejecutivos del WTC que entrevistamos, es dispersar las operaciones. «¿Vamos a tener una sola operación, un solo sitio en la ciudad de Nueva York?», preguntaba un ejecutivo. «La respuesta es, claramente, no, no vamos a...», fue su contestación. Otros ejecutivos del WTC relacionaron dispersión y seguridad más explícitamente:

«Número uno, hemos repensado decididamente nuestra estrategia de tener todas las aplicaciones y funciones relativas a misiones decisivas, sean electrónicas o humanas, en una única ubicación, y aquélla no se limita precisamente a los edificios, sino a la geografía».

\footnotetext{
12 En su análisis de los mercados de capital alemanes, Lo y Grote (2001) han llegado separadamente a una conclusión parecida. Los autores sostienen que «con la virtualización de las bolsas, los excedentes con otros traders y la oficina central de las empresas se convierten en los principales determinantes de la localización de los traders» (Lo y Grote, 2001, p. 2). Por ejemplo, la bolsa Eurex, en Frankfurt, se benefició de la tecnología más nueva para atraer al Bundt alemán desde Londres. Pero el conocimiento requerido por las operaciones siguió estando situado en las comunidades de traders de Londres. A consecuencia de ello, el Bundt alemán se negocia en Frankfurt, pero los traders siguen estando localizados en Londres.
} 
Cuando los ejecutivos consideran las decisiones para dispersar sus operaciones no sólo a través de los edificios, sino también a través de las redes eléctricas, códigos telefónicos y centros de transporte, se enfrentan a nuevos desafíos. Por una parte, la innovación eficaz, y en particular el tipo de innovación que contribuye a realizar operaciones rentables, requieren proximidad a los otros departamentos de la misma empresa y de las empresas rivales. Por otra, los intereses de la seguridad en cuanto a ataques potenciales requieren que las empresas dispersen sus operaciones. En consecuencia, las empresas financieras están atrapadas entre el tirón de la proximidad para la innovación y el impulso de la dispersión para la seguridad ${ }^{13}$. Uno de los ejecutivos del Trade Center que entrevistamos expresó esta preocupación:

«Por tanto, cuando pienso en la medición del estado de preparación, una de las cosas que (...) oí alto y claro esta mañana de nuevo es la capacidad de la organización para operar geográficamente dispersa, eficazmente, y éstos son dos conceptos muy distintos que tienen que existir mutuamente».

La tendencia hacia la dispersión pone a prueba tanto a los responsables de las políticas como a los ejecutivos. En el proceso de dispersión, las empresas inevitablemente reubicarán algunas de sus partes fragmentadas del espacio de oficinas más allá del Bajo Manhattan. ¿Cómo responderán a esta dinámica los responsables de las políticas, actualmente preocupados con la reconstrucción de la Zona Cero y el Bajo Manhattan?

Una respuesta obvia para los responsables de las políticas preocupados con la viabilidad del distrito es replicar lo que estaba allí antes de la destrucción del once de septiembre, incluyendo la estricta identificación del Bajo Manhattan con Wall Street. Construir unas nuevas torres (dos o veinte) exactamente con la misma superficie de espacio para oficinas y esperar que las empresas financieras vuelvan. La Empire State Development Corporation (ESDC), por ejemplo, parece dar por sentado que los intereses de Wall Street y del Bajo Manhattan estarán mejor servidos manteniendo las empresas financieras donde están ahora. El organismo público ha creado un programa de subvenciones personalizadas, negociado individualmente en el caso de grandes empresas, para frenar la corriente de empresas que abandonan la zona. Y cada vez que una de estas empresas decide marcharse del Bajo Manhattan, la ESDC toma la decisión como una derrota.

\footnotetext{
13 Amin y Cohendet (2003) señalan una salida a este impasse de las empresas financieras: la capacidad de la organización para utilizar espacios distintos del físico para transferir conocimiento. La organización tiene, según los autores, mecanismos que se parecen a «las comunidades de entusiastas con intereses semejantes (por ejemplo, vegetarianos, grupos de bricolaje, manifestantes callejeros, psicólogos clínicos) que se mantienen colectivamente por los viajes baratos, Internet y la literatura especializada. Incluye las comunidades de la diáspora basadas en relaciones migratorias y afiliaciones y conexiones transnacionales y culturales» (Amin y Cohendet, 2003, p. 128).
} 
Pero en este punto cabe preguntarnos si los intereses del Bajo Manhattan están realmente mejor servidos manteniendo su identificación, de antes del once de septiembre, con Wall Street y los problemas relacionados con un horario de nueve a cinco, con calles desiertas al anochecer, actividad comercial en declive y vida nocturna deprimente. Evitando que las empresas financieras se marchen y liberando espacio para nuevos arrendatarios, las subvenciones impiden la evolución del área, agravando sus defectos en lugar de abordarlos correctamente. A su vez, esta voluntad pide subvenciones renovadas.

Contrariamente, la estrategia de las políticas públicas consiste en prever cómo serán las finanzas dentro de veinte o treinta años, e intentar diseñarlas de forma correspondiente. El problema que presenta este planteamiento se hace evidente cuando reflexionamos sobre la revolución en las finanzas cuantitativas que se ha dado en el sector. Hace treinta años, el Trade Center se estaba construyendo como un «puerto vertical» que albergaría y promovería las tradicionales empresas comerciales ubicadas en el puerto de Nueva York: empresas que compraban mercaderías como cacao en un continente y lo transportaban y vendían en otro. Esta medida tuvo un claro espíritu previsor (dirigió la transición de una economía industrial a una economía de servicios) y, a pesar de ello, nadie preveía que el Trade Center fuera un centro de operaciones financieras. $Y$ es probable que nadie haya predicho que las finanzas pasarían por las tres etapas de la revolución cuantitativa. La fórmula de BlackScholes para el cálculo de los precios de los derivados (una de las aplicaciones clave de las matemáticas a las finanzas) se desarrolló en 1973 y, en su momento, casi ni apareció en la pantalla de radar de los responsables de las políticas públicas. En 1973, los ordenadores eran artefactos del tamaño de una habitación y se utilizaban para procesar nóminas. Y hablar de la World Wide Web y de «conectividad con Internet de banda ancha» en 1973 podría haber provocado la sospecha de que el contacto de uno con la realidad estaba afectado por demasiadas dosis de drogas. En resumen, la revolución cuantitativa en las finanzas habría sido difícil de prever, y más difícil aún diseñar políticas acordes a ella. Con esta retrospección como advertencia, ¿quién puede decir con suficiente confianza cómo serán las finanzas dentro de treinta años?

En lugar de predecir el futuro o de replicar el pasado reciente, los ciudadanos de Nueva York deberían animar a sus representantes a reconstruir el Bajo Manhattan poniendo énfasis en la creciente diversidad de las organizaciones; no simplemente en una mayor cantidad de grandes corporaciones, sino empresas de tamaño medio y start-ups; no simplemente servicios financieros, sino una amplia gama sectorial; no simplemente negocios, sino instituciones educativas y culturales. La diversidad cumple dos tareas. Primera, hace del Bajo Manhattan un escenario más vibrante y emocionante, y por eso más atractivo para empresas intensivas en conocimiento que serán una fuente de vitalidad económica para la ciudad. ¿Por qué viene a Nueva York la gente activa, ambiciosa y joven? Porque otras per- 
sonas jóvenes, ambiciosas y activas como ellas también vienen aquí. Cuanto más sea, una ciudad o un distrito de una ciudad, un lugar para asombrarse y emocionarse, más puede estimular estos efectos puntuales. Segunda, una mayor diversidad entre los tipos de organizaciones produce una reserva de genes más amplia, a partir de la cual pueden surgir nuevas combinaciones innovadoras.

¿Vendrá tal estrategia del propio sector financiero? Como las estrategias generativas -con sus relaciones laterales, heterárquicas - que se mostraron tan elásticas y eficaces en los primeros días y semanas de la recuperación, una estrategia generativa para reconstruir el Bajo Manhattan requerirá relaciones horizontales más amplias, que impliquen activamente a los ciudadanos y a las asociaciones cívicas. Al igual que las propias salas de operaciones, las nuevas asociaciones que contribuyan a la innovación requerirán un acceso a la información repartido y la organización de la diversidad.

\section{AGRADECIMIENTOS}

Se presentó una primera versión de este artículo como Keynote Lecture en la reunión anual de la Society for the Advancement of Socio-Economics, en Minneapolis, el 28 de junio de 2002. La investigación en la que se basa el mismo fue financiada mediante una beca de la National Science Foundation (NSF IIS 0233489) y una beca de la Russell Sage Foundation. Damos las gracias a Monique Girard, Gernot Grabher, Alex Hicks, Fabian Muniesa, Gina Neff y a los dos evaluadores anónimos de la Socio-Economic Review por sus comentarios y sugerencias a un primer borrador.

\section{REFERENCIAS}

ABOLAFIA, M. Y. (1996): Making Markets: Opportunism and Restraint on Wall Street, Cambridge, MA, Harvard University Press.

ALLEN, T. (1977): Managing the Flow of Technology, Cambridge, MA, MIT Press.

AMIN, A., y COHENDET, P. (2003): Knowledge, Practices, Communities, and Competences in Firms, Oxford, Oxford University Press, en curso de publicación.

AMIN, A., y THRIFT, N. (1992): «Neo-Marshallian Nodes in Global Networks», International Journal of Urban and Regional Research, 16, 571-587.

ANÓNIMO (2001): «The Markets Rewired», The Economist, 22 de septiembre, pp. 68-69.

BAGLI, C. V. (2002): «Downtown, An Exodus That Cash Can’t Stop», The New York Times, 24 de julio.

BERMAN, D. (2002): «Will They Return?: Wall Street's Diaspora After September $11^{\text {th }}$, Centre on Organizational Innovation, Columbia University. 
BEUNZA, D., y STARK, D. (2002): «Tools of the Trade: The Socio-Technology of Arbitrage in a Wall Street Trading Room», Conference on Social Studies of Finance, Nueva York, NY, Social Science Research Council, 3-4 de mayo.

BROWN, J. S. (1997): «Introduction: Rethinking Innovation in a Changing World», en J. S. Brown (ed.), Seeing Differently: Insights on Innovation, Boston, MA, Harvard Business School Press, pp. ix-xxviii.

BROWN, J. S., y DUGUID, P. (1998): «Organizing Knowledge», California Management Review, 401, 90-111.

- (2000): The Social Life of Information, Boston, MA, Harvard Business School Press.

- (2001): «Knowledge and Organization: a Social-Practice Perspective», Organization Science, 12, $198-213$.

CALLON, M. (1998): «Introduction: The Embeddedness of Economic Markets in Economics», en M. Callon (ed.), The Laws of the Markets, Oxford, Blackwell Publishers.

CALLON, M., y MUNIESA, F. (2002): «Economic Markets as Calculative and Calculated Collective Devices», manuscrito, París, Centre de Sociologie de l'Innovation, École des Mines de Paris.

CASTELLS, M. (1996): The Rise of the Network Society, Cambridge, MA, Blackwell Publishers.

CLARK, G. L.; FELDMAN, M. P., y GERTLER, M. S. (2000): The Oxford Handbook of Economic Geography, Oxford, Oxford University Press.

CLIPPINGER, J. H. (1999): «Tags: The Power of Labels in Shaping Markets and Organizations», en J. H. Clippinger (ed.), The Biology of Business: Decoding the Natural Laws of Enterprise, San Francisco, CA, Jossey-Bass, pp. 67-88.

CORBRIDGE, S., y THRIFT, N. (1994): «Introduction and Overview», en S. Corbridge y N. Thrift (eds.), Money, Power and Space, Londres, Blackwell Publishers.

DiMAGGIO, P. (ed.) (2001): The Twenty-First-Century Firm: Changing Economic Organization in International Perspective, Princeton, NJ, Princeton University Press.

DUNBAR, N. (2000): Inventing Money: The Story of Long-term Capital Management and the Legends Behind It, Nueva York, NY, John Wiley \& Sons.

FLYNN, K.; WORTH, R. F.; PRISTIN, T.; GOOTMAN, E.; ZHAO, Y.; BAYOT, J., y O'DONNELL, M. (2002): «For Towers' Refugee Businesses, a Year of Struggle and Change», The New York Times, 11 de septiembre, p. 25.

GALISON, P. L. (1997): Image and Logic: a Material Culture of Microphysics, Chicago, IL, University of Chicago Press. GALISON, P. L., y THOMPSON, E. (eds.) (1999): The Architecture of Science, Cambridge, MA, MIT Press.

GIRARD, M., y STARK, D. (2002): «Distributing Intelligence and Organizing Diversity in New Media Projects», Environment and Planning A, 34, 1927-1949.

GLADWELL, M. (2000): «Designs for Working», The New Yorker, 11 de diciembre, pp. 60-70.

GRABHER, G. (1994): In Praise of Waste: Redundancy in Regional Development, Berlín, Edition Sigma.

- (2002a): «Cool Projects, Boring Institutions: Temporary Collaboration in Social Context», Regional Studies, 36, 205-214.

— (2002 b): «The Project Ecology of Advertising: Tasks, Talents and Teams», Regional Studies, 36, 245-262.

GRABHER, G., y STARK, D. (1997): «Organizing Diversity: Evolutionary Theory, Network Analysis, and the Postsocialist Transformations", en G. Grabher y D. Stark (eds.), Restructuring Networks in Postsocialism: Legacies, Linkages, and Localities, Nueva York, NY, y Londres, Oxford University Press, pp. 1-32. 
GROTE, M.; LO, V., y HARRSCHAR-EHRNBORG, S. (2002): «A Value Chain Approach to Financial Centres - The Case of Frankfurt», Tijdschrift voor Economische en Sociale Geografie, 93, 412-423.

HEATH, C.; JIROTKA, M.; LUFF, P., y HINDMARSH, J. (1995): «Unpacking Collaboration: the Interactional Organization of Trading in a City Dealing Room», Computer Supported Cooperative Work, 3, 147-165.

HUTCHINS, E. (1995): Cognition in the Wild, Cambridge, MA, MIT Press.

HUTCHINS, E., y KLAUSEN, T. (1991): «Distributed Cognition in an Airline Cockpit», en Y. Engestrom y D. Middleton (eds.), Distributed Cognition and Communication at Work, Cambridge, Cambridge University Press.

KIM, S. (1991): «Heterogeneity of Labor Markets and City Size in an Open Spatial Economy», Regional Science and Urban Economics, 21, 109-126.

KELLY, J., y STARK, D. (2002): «Crisis, Recovery, Innovation: Learning from 9/11», Environment and Planning A, 34 (septiembre), 1523-1533.

KNORR CETINA, K., y BRUEGGER, U. (2002): “Global Microstructures: the Virtual Societies of Financial Markets», American Journal of Sociology, 107, 905-950.

KOGUT, B., y ZANDER, U. (1992): «Knowledge of the Firm, Combinative Capabilities, and the Replication of Technology», Organization Science, 5, 383-397.

KRAUT, R. E.; FUSSELL, S. R.; BRENNAN, S. E., y SIEGEL, J. (2002): en P. Hinds y S. Kiesler (eds.), Distributed Work, Cambridge, MA, MIT Press, pp. 137-162.

LANE, D., y MAXFIELD, R. (1996): «Strategy under Complexity: Fostering Generative Relationships», Long Range Planning, 29, 215-231.

LATOUR, B. (1991): «Technology is Society Made Durable», en J. Law (ed.), A Sociology of Monsters: Essays on Power, Technology, and Domination, Londres y Boston, MA, Routledge \& Kegan Paul, pp. 103-131.

LEPINAY, V. (2002): «Finance as Circulating Formulas», texto presentado en la New York Conference on Social Studies of Finance, Nueva York, NY, Columbia University, mayo.

LEPINAY, V., y ROUSSEAU, F. (2000): «Les trolls sont-ils incompétents? Enquête sur les financiers amateurs», Politix, 13 (52), 73-97.

LEWIS, M. (1999): «How the Eggheads Cracked», The New York Times, 24 de enero.

LEYSHON, A., y THRIFT, N. (1997): Money/Space: Geographies of Monetary Transformation, Londres y Nueva York, NY, Routledge.

LO, V., y GROTE, M. (2001): «Where Traders Go When Stock Exchanges Go Virtual - Concentration, Dissemination or Persistence?», en M. Balling, F. Lierman y A. Mullineux (eds.), Technology and Finance. Challenges for Financial Markets, Business Strategies and Policy Makers, Londres, Routledge.

MacKENZIE, D. (2000): «Long-Term Capital Management and the Sociology of Finance», The London Review of Books, 13 de abril.

MacKENZIE, D., y MILLO Y.: «Constructing a Market, Performing Theory: TheHistorical Sociology of a Financial Derivatives Exchange», American Journal of Sociology, July 2003, 109 (1), 107-145.

MILLO, Y. (2001): «Safety in Numbers: How Exchanges and Regulators Shaped Index-Based Derivatives», texto presentado en la New York Conference on Social Studies of Finance, Nueva York, NY, Columbia University, mayo.

MUNIESA, F. (2000): «Un robot walrasien. Cotation électronique et justesse de la découverte des prix», Politix, 13, 121-154.

- «Reserved Anonymity: on the Use of Telephones in the Trading Room», texto presentado en la New York Conference on Social Studies of Finance, Nueva York, NY, Columbia University, mayo. 
NEFF, G., y STARK, D. (2003): «Permanently Beta: Responsive Organization in the Internet Era», en P. E. N. Howard y S. Jones (eds.), Society Online: The Internet in Context, Thousand Oaks, CA, Sage, en curso de publicación.

OLSON, J. S.; TEASLEY, S.; COVI, L., y OLSON, G. (2002): «The (Currently) Unique Advantages of Collocated Work», en P. Hinds y S. Kiesler (eds.), Distributed Work, Cambridge, MA, MIT Press, pp. 113-135.

ORLIKOWSKI, W. J. (2002): «Knowing in Practice: Enacting a Collective Capability in Distributed Organizing», Organization Science, 13, 249-273.

PORTEUS, D. (1999): «The Development of Financial Centers: Location, Information Externalities and Path Dependence», en R. Martin (ed.), Money and the Space Economy, Chichester, John Wiley \& Sons, pp. 95-114.

POWELL, W. W. (1996): «Inter-organizational Collaboration in the Biotechnology Industry», Journal of Institutional and Theoretical Economics, 152, 197-215.

POWELL, W. W., y OWEN-SMITH, J. (1998): «Universities and the Market for Intellectual Property in the Life Science», Journal of Policy Analysis and Management, 17, 253-277.

PREDA, A. (2002): «On Ticks and Tapes: Financial Knowledge, Communicative Practices, and Information Technologies on $19^{\text {th }}$ Century Financial Markets", texto presentado en la New York Conference on Social Studies of Finance, Nueva York, NY, Columbia University, mayo.

SMITH, C. (1990): Auctions: The Social Construction of Value, Berkeley, CA, University of California Press.

SORKIN, M., y ZUKIN, S. (eds.): After the World Trade Center: Rethinking New York City, Nueva York, NY, y Londres, Routledge.

STARK, D. (1999): «Heterarchy: Distributing Intelligence and Organizing Diversity», en J. Clippinger (ed.), The Biology of Business: Decoding the Natural Laws of Enterprise, San Francisco, CA, Jossey-Bass, pp. 153-179.

- (2000): «For a Sociology of Worth», discurso de apertura, Annual Conference of the European Association of Evolutionary Political Economy, Berlín, 3 de noviembre.

SUCHMAN, L. (1987): Plans and Situated Actions: the Problem of Human-Machine Communication, Nueva York, NY, Cambridge University Press.

THRIFT, N. (1994): «On the Social and Cultural Determinants of International Financial Centres: the Case of the City of London», en S. Corbridge, N. J. Thrift y R. L. Martin (eds.), Money, Power and Space, Oxford, Blackwell, pp. 327-355.

- (2000): «Pandora's Box? Cultural Geographies of Economies», en G. L. Clark, M. P. Feldman y M. S. Gertler (eds.), The Oxford Handbook of Economic Geography, Oxford, Oxford University Press, pp. 689-704.

VAN DEN BULTE, C., y MOENAERT, R. K. (1998): «The Effect of R\&D Team Co-Location on Communication Patterns among R\&D, Marketing and Manufacturing», manuscrito, Pensilvania, PA, Marketing Department, The Wharton School, University of Pennsylvania.

WENGER, E. (1998): Communities of Practice: Learning, Meaning, and Identity, Cambridge, Cambridge University Press.

WHITE, H., y MISCHE, A. (1998): «Between Conversation and Situation: Public Switching Dynamics across Network Domains», Social Research, 65, 695-724.

WOLFE, T. (1987): The Bonfair of the Vanities, Nueva York, NY, Farrar, Straus and Giroux.

ZAHEER, S. A. (1997): «Acceptable Risk: A Study of Global Currency Trading Rooms in the US», working paper, Wharton Financial Institutions Center, pp. 97-122.

ZALOOM, C. (2002): «Ambiguous Numbers: Trading and Technologies in Global Financial Markets», texto presentado en la New York Conference on Social Studies of Finance, Nueva York, NY, Columbia University, mayo. 


\section{ABSTRACT}

What is the organizational basis of the capacity for recovery from a crisis? In this study, we examine a financial operations room that was damaged in the attack on the World Trade Center on $11^{\text {th }}$ September 2001. What does the crisis reveal about social practices and the technological instruments of buying and selling? Taking as the point of departure our field research carried out prior to the eleventh of September, we demonstrate how the heterarchical (as opposed to the hierarchical) organization of the operations room spontaneously stimulated innovations, as events developed. Through the observations we have made after the attack, as well as approach groups with executives from various World Trade Center financial companies, we show that the heterarchical characteristics contributed to innovation in the response to the crisis. Under conditions of radical uncertainty, it is not possible to know beforehand about the resources that will be necessary, or even know in advance what a resource could be. Laterally distributed intelligence and the tolerance of multiple appraisal and interaction registers provide generative structures in which the replicative redundancy of planning for emergencies faces its limits. We conclude with a brief discussion of the implications that our findings on innovation, location and capacity for response have for present-day changes in the urban geography of finance in Lower Manhattan.

Key words: Theory of Organizations, Heterarchy, Planning for Emergencies, Socio-technical Networks, Economic Geography, Eleventh of September [2001]. 